\title{
A note on the existence of Nash networks in one-way flow models
}

\author{
Jean Derks • Martijn Tennekes
}

Received: 5 June 2008 / Accepted: 13 August 2008 / Published online: 3 September 2008

(C) The Author(s) 2008. This article is published with open access at Springerlink.com

\begin{abstract}
We study the one-way flow model of network formation with ownerhomogeneous link costs and heterogeneous profits. Recently, several proofs of existence of Nash networks are discussed in literature. The proof by Billand et al. (Econ Theory, 2007, forthcoming) is based on a nice and clear idea, but the technical elaboration is tedious and too complex in our opinion. In this note, we provide an alternative and easy accessible proof based on the same idea. Also, we show by means of a counterexample that Nash networks may not exist for games where link costs are heterogeneous, but arbitrarily close to owner-homogeneity.
\end{abstract}

Keywords Network formation · Non-cooperative games

JEL Classification $\quad$ C72 $\cdot$ D85

\section{Introduction}

Bala and Goyal (2000) introduce a non-cooperative game model of network formation. Agents, who correspond to the nodes of the network, are able to form links unilaterally, i.e. without consent of other agents. Agents receive profits from being connected to other agents. However, links are expensive. Two variants are being studied by

We would like to thank an anonymous referee for useful comments.

\footnotetext{
J. Derks · M. Tennekes $(\varangle)$

Department of Mathematics, Maastricht University, P.O. Box 616, $6200 \mathrm{MD}$, Maastricht, The Netherlands

e-mail: martijn.tennekes@micc.unimaas.nl

J. Derks

e-mail: jean.derks@micc.unimaas.nl
} 
Bala and Goyal (2000): the one-way flow model and the two-way flow model. Profits flow along the direction of the arcs in the one-way flow model, while they flow along both directions of the arcs in the two-way flow model. In this note, we study the oneway flow model. The two-way flow model has been studied by Galeotti et al. (2006), Haller and Sarangi (2005), and Haller et al. (2007).

For the one-way flow model, the architecture of Nash networks has been addressed by Bala and Goyal (2000) and Galeotti (2006). Bala and Goyal (2000) prove the existence of Nash networks when link costs and profits are homogeneous, i.e. all links are equally expensive, and each agent receives a constant profit value for being connected to each other agent.

The existence of Nash networks in one-way flow models for owner-homogeneous link costs, i.e. all links are equally expensive with respect to the owner, and heterogeneous profits has been proved by Billand et al. (2007) and independently by Derks et al. (2008a). These two proofs are totally different from each other. The latter is a proof by induction; to show that a Nash network exists for a game with $n$ agents, Derks et al. (2008a) used the induction hypothesis that a Nash network exists for each game with less than $n$ agents. Billand et al. (2007) provide a constructive proof, in which a sequence of networks is constructed where each successive network is as least as good for each agent. The idea behind their approach is very nice and clear. However, its implementation is complicated and too technical in our opinion. Therefore, we provide an alternative proof in this note, that is directly based on the same idea.

Furthermore, Billand et al. (2007) provide conditions for the existence of Nash networks when link costs are heterogeneous. According to these conditions, Nash networks exist when the difference between any two link costs is smaller than the constant profit value. In this note, we show by means of a counterexample that this result is not correct. For this counterexample, where link costs are arbitrarily close to the situation of owner-homogeneity, we show that Nash networks do not exist.

A different approach to study the existence of Nash networks is used by Derks et al. (2008b). They model network formation as a dynamic game in which agents play local actions, which are adding, removing or replacing a link. Furthermore, they study payoff functions that fulfill a specified framework of properties. By a series of improving local actions, they prove the existence of local-Nash networks, which are global-Nash as well.

\section{Model and notations}

Let $N=\{1, \ldots, n\}$ denote a finite set of agents. We define a one-way flow network $g$ on the agent set $N$ as a set of links $g \subseteq N \times N$, where loops are not allowed, i.e. $(i, i) \notin g$ for all $i \in N$.

We say that a link $(j, i)$, which is directed to $i$, is owned by $i$. Let $g_{-i}$ denote the network obtained from $g$ after removing the links owned by $i$.

A directed path from $j$ to $i$ in $g$ is a sequence of distinct agents $i_{1}, i_{2}, \ldots, i_{k}$ with, $k \geq 1$, such that $j=i_{1}, i=i_{k}$ and $\left(i_{s}, i_{s+1}\right) \in g$ for each $s=1,2, \ldots, k-1$. An undirected path is defined analogously, but here, either $\left(i_{s}, i_{s+1}\right)$ or $\left(i_{s+1}, i_{s}\right)$ is contained in $g$ for each $s=1,2, \ldots, k-1$. 
Let $\operatorname{Car}(g)$, the carrier of network $g$, be the set of agents who are begin- or endpoints in $g$. For a network $g$ we define $g^{j}$, the component of $g$ that contains agent $j$, as the network containing all links that are connected to $j$ by some undirected path. Since $g^{j}$ may be empty, which is the case when $j$ is isolated in $g$, we assume that $j$ is contained in the carrier of $g^{j}$, i.e. let $j \in \operatorname{Car}\left(g^{j}\right)$.

Let $N_{i}(g)=\{j \in N$ : a directed path from $j$ to $i$ exists in $g\}$. We will refer to the agents in this set as observed agents by agent $i$ in network $g$. Let $N_{i}^{d}(g)=\{j:(j, i) \in g\}$. Note that $i \in N_{i}(g)$, and $i \notin N_{i}^{d}(g)$.

For each agent $i$, let $\pi_{i}: \mathcal{G} \rightarrow \mathbb{R}$ be a payoff function, where $\mathcal{G}$ is the set of all possible one-way flow networks. We will use the following payoff function, which has been proposed by Galeotti (2006).

$$
\pi_{i}(g)=\sum_{j \in N_{i}(g)} v_{i j}-\sum_{j \in N_{i}^{d}(g)} c_{i j}
$$

Here, $v_{i j}$ is the profit that agent $i$ receives from being connected to $j$ and $c_{i j}$ is the cost of link $(j, i)$ for agent $i$. Thus, agent $i$ pays for each own link, i.e., for each link ending at $i$, and he receives profits from being connected to each other agent $j$, which is the case when a directed path from $j$ to $i$ exists.

We say that link costs are homogeneous if there is a constant $c$ with $c_{i j}=c$ for all $i, j \in N$. Link costs are owner-homogeneous if for each agent $i$ there is a constant $c_{i}$ with $c_{i j}=c_{i}$ for all $j \in N$. Otherwise, the link costs are heterogeneous. These definitions also apply to the profits. The profits and link costs are assumed to be non-negative throughout this paper.

In this paper we study a non-cooperative game. This game is played by the agents in $N$. Simultaneously and independently, each agent $i$ chooses a, possibly empty, set $S$ of agents he wants to connect to by creating the links $(j, i)$, for each $j \in S$. Together, the links of all agents form a network $g \in \mathcal{G}$. Then, each agent $i$ receives a payoff $\pi_{i}(g)$.

We define an action of agent $i$ to a network $g$ by a set of agents $S \subseteq N \backslash\{i\}$. The network, after $i$ chooses to link up with the agents in $S$, is described by

$$
g_{-i} \cup\{(j, i): j \in S\} .
$$

An action $S^{*}$ of agent $i$ is called a best response if

$$
\pi_{i}\left(g_{-i} \cup\left\{(j, i): j \in S^{*}\right\}\right) \geq \pi_{i}\left(g_{-i} \cup\{(j, i): j \in S\}\right)
$$

for all actions $S \subseteq N \backslash\{i\}$. A network $g$ is a Nash network if $N_{i}^{d}(g)$ is a best response for all $i \in N$, i.e., if for each agent $i$

$$
\pi_{i}(g) \geq \pi_{i}\left(g_{-i} \cup\{(j, i): j \in S\}\right)
$$

for all actions $S \subseteq N \backslash\{i\}$ 
Let $g_{-i j}=g_{-i}^{j} \cup\{(j, i)\}$. Recall that $g_{-i}$ is network $g$ where all $i$ 's links are removed, so that $g_{-i}^{j}$ is the component of $g_{-i}$ where $j$ is contained in its carrier. It is immediate that

$$
\pi_{i}(g) \leq \pi_{i}(g \backslash\{(j, i)\})+\pi_{i}\left(g_{-i j}\right)
$$

when link $(j, i)$ is contained in $g$. Therefore, when agent $i$ plays a best response then $\pi_{i}\left(g_{-i j}\right) \geq 0$ for each $j \in N_{i}^{d}(g)$. We say that link $(j, i)$ is beneficial in $g$ when $\pi_{i}\left(g_{-i j}\right) \geq 0$, and a network $g$ is called beneficial when all its links are beneficial. Observe that a Nash network is beneficial.

\section{Non-existence of Nash networks}

Billand et al. (2007) claim that Nash networks always exist if for all $i, j, j^{\prime} \in N$ holds that $\left|c_{i j}-c_{i j^{\prime}}\right|<v_{i}$ (see their Proposition 3). This is not true due to the following example.

Example 1 (Derks et al. 2008a) Let $n=4$ and call the agents 1, 2, 3 and 4. Let $\pi$ be a payoff function defined by (1), where link costs are heterogeneous and profits are homogeneous and normalized to 1 . The numbers next to the links in Fig. 1 indicate the costs of these links. Here, $\epsilon$ is a strictly positive number which can be chosen arbitrarily close to 0 . The costs of the links that are not depicted in this figure are the following:

- links owned by agent 1 have costs $1+\epsilon$,

- links owned by agent 2 have costs $2+\epsilon$,

- links owned by agents 3 and 4 have costs $3+\epsilon$,

Notice that since $v_{i i}=1, \pi_{i}\left(g_{-i}\right)=1$ for any network $g$ and any agent $i$.

The best response of agent 4 to any network is either $\{2\}$ or $\varnothing$, since those are the only actions for which agent 4 might have a payoff of at least 1 . First, suppose that agent 4 plays $\{2\}$ as a best response in a Nash network. Consequently, the unique best response of agent 1 is $\{4\}$. Agent 2 has one unique best response to this situation: $\{1\}$. Finally, agent 3 has one unique best response, which is $\{2\}$. The obtained network is the same as depicted in Fig. 1. It follows that $\{2\}$ is not a best response of agent 4 , since $\emptyset$ gives a higher payoff. This contradicts our assumption. Hence, there is no Nash network in which agent 4 plays $\{2\}$.

Fig. 1 The link costs

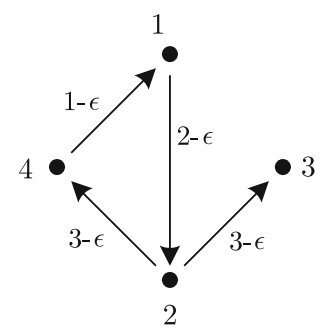


Fig. 2 Network obtained in

Example 1

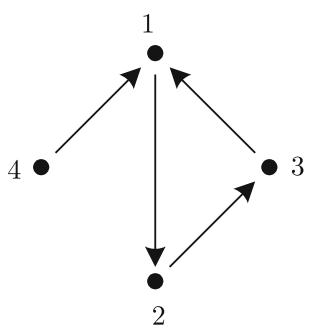

Now suppose that agent 4 plays $\emptyset$ as a best response in a Nash network. Agent 1 will include 4 in every best response to this situation. Then, the unique best response for agent 2 is $\{1\}$. To this situation, the unique best response of agent 3 is $\{2\}$. Hence, the unique best response of agent 1 is $\{3,4\}$ (see Fig. 2). Now agent 4 has a unique best response to this new situation, which is $\{2\}$. This contradicts our assumption of agent 4 playing $\emptyset$ in a Nash network. Hence no Nash networks exist.

For this example, link costs are close to owner-homogeneity, i.e. $\left|c_{i j}-c_{i j^{\prime}}\right| \leq 2 \epsilon$ for all $i, j, j^{\prime} \in N$. Since $v_{i j}=1$ for all $i, j \in N$, and since $\epsilon$ can be chosen arbitrarily small to 0 , we have $\left|c_{i j}-c_{i j^{\prime}}\right|<1$. Therefore this example is a counterexample for Proposition 3 by Billand et al. (2007).

\section{Existence of Nash networks}

In this section, we prove the existence of Nash networks for games with ownerhomogeneous link costs, i.e. $c_{i j}=c_{i}$ for all $i, j \in N$.

Let a network be proper if the outdegree of each agent is at most 1 . In a proper network $g$, an agent $i$ observes each agent $j \in N_{i}(g)$ via a unique directed path, and by this observation it is not hard to see that equality holds in (2), whenever $g$ is proper.

For any network $g$ let $C(g)$ denote the set of agents located on a directed cycle in $g$. Further, let $D(g)$ denote the set of agents who observe at least one agent in $C(g)$, i.e. $D(g)=\left\{i: N_{i}(g) \cap C(g) \neq \emptyset\right\}$.

Assuming that the agents are numbered $1,2, \ldots, n$ there is a unique ordering $s_{1}, s_{2}, \ldots, s_{d}$ of agents in $D(g)$, with $s_{k}<s_{k+1}$ for each $k=1,2, \ldots, d=|D(g)|$. Consider the following network $\hat{g}$, defined by $\hat{g}=g$ in case $d=0$, and for $d \geq 2$ (notice that $d=1$ does not exist, since a cycle contains at least two agents):

$$
\hat{g}=\left\{\left(s_{1}, s_{2}\right), \ldots,\left(s_{d-1}, s_{d}\right),\left(s_{d}, s_{1}\right)\right\} \cup\{(j, i) \in g: j \notin D(g)\} .
$$

An example of this network is depicted in Fig. 3.

Lemma 1 If for a network $g$ the agents outside $D(g)$ have at most one outgoing link then $\hat{g}$ is proper.

Proof Each agent in $D(g)$ has exactly one outgoing link in $\hat{g}$, namely the one on the cycle. Each agent outside $D(g)$ has the same outdegree in $\hat{g}$ as in $g$. 


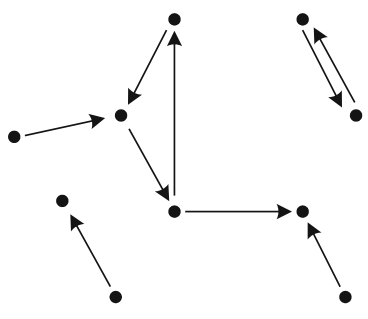

$g$

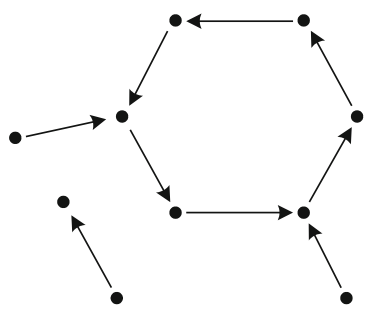

$\hat{g}$

Fig. 3 Network $g$ with the corresponding network $\hat{g}$

Observe that $g=\hat{g}$ whenever $D(g)=\emptyset$, i.e. whenever there are no cycles in $g$. Otherwise, $\hat{g}$ has exactly one cycle. Notice also that an agent has no more links in $\hat{g}$ than in $g$, so that in the owner-homogeneous costs situation the agents in $\hat{g}$ face costs at most as in $g$. Furthermore, the agents observe the same or more agents in $\hat{g}$ :

Lemma $2 N_{i}(g) \subseteq N_{i}(\hat{g})$ for each agent $i$.

Proof Let $j \in N_{i}(g)$. Suppose $i \notin D(g)$, then any directed path from $j$ to $i$ in $g$ does not visit an agent in $D(g)$, and therefore it is also a directed path in $\hat{g}$. This implies that $i$ observes $j$ in $\hat{g}: j \in N_{i}(\hat{g})$.

Now suppose $i \in D(g)$. When also $j \in D(g)$ then $i$ observes $j$ via the cycle in $\hat{g}$. So, let $j \notin D(g)$, and let $k$ be the first agent in $D(g)$ on a directed path from $j$ to $i$ in $g$. Then, the subpath from $j$ to $k$ is also a directed path in $\hat{g}$, implying $j \in N_{k}(\hat{g})$. Since $k \in N_{i}(\hat{g})$, we must have $j \in N_{i}(\hat{g})$.

Lemma 3 Let the payoff function be given by (1) where link costs are ownerhomogeneous, i.e. $c_{i j}=c_{i}$ for all $i, j \in N$. If a network $g$ is beneficial, then network $\hat{g}$ is also beneficial.

Proof Let $(j, i) \in \hat{g}$. We prove that $(j, i)$ is beneficial in $\hat{g}$.

Suppose $j \notin D(g)$. This implies $(j, i) \in g$. For $k \in N_{i}\left(g_{-i j}\right)$ there is a directed path from $k$ to $i$ via link $(j, i)$ in $g$, and none of the visited agents are members of $D(g)$ (except possibly $i$ ), so that this path is also present in $\hat{g}_{-i j}$. This implies $k \in N_{i}\left(\hat{g}_{-i j}\right)$, and therefore,

$$
\pi_{i}\left(\hat{g}_{-i j}\right)=\sum_{k \in N_{i}\left(\hat{g}_{-i j}\right)} v_{i k}-c_{i} \geq \sum_{k \in N_{i}\left(g_{-i j}\right)} v_{i k}-c_{i}=\pi_{i}\left(g_{-i j}\right) .
$$

Beneficiality of link $(j, i)$ in $g$ implies therefore the beneficiality of $(j, i)$ in $\hat{g}$.

Now, suppose $j \in D(g)$. Then the link $(j, i)$ is a link of the cycle of $\hat{g}$. It is evident that $D(g) \subseteq N_{i}\left(\hat{g}_{-i j}\right)$. Also, $D(g) \cap N_{i}^{d}(g) \neq \emptyset$, say $k \in D(g) \cap N_{i}^{d}(g)$. We know that $(k, i)$ is beneficial in $g$. Since link costs are owner-homogeneous, i.e. $c_{i k}=c_{i j}$, we conclude that link $(j, i)$ is beneficial in $\hat{g}$ whenever

$$
N_{i}\left(g_{-i k}\right) \subseteq N_{i}\left(\hat{g}_{-i j}\right)
$$


So, let $r \in N_{i}\left(g_{-i k}\right)$. If $r \in D(g)$ then also $r \in N_{i}\left(\hat{g}_{-i j}\right)$ as we mentioned before. Thus, assume $r \notin D(g)$. There is a directed path from $r$ to $k$ in $g_{-i}$. Let $r^{\prime}$ be the first agent from $D(g)$ on this path. Then the subpath from $r$ to $r^{\prime}$ is present in $\hat{g}$. Extend this path with the directed path from $r^{\prime}$ to $j$ on the cycle of $\hat{g}$, thus obtaining a directed path from $r$ to $j$ in $\hat{g}$. This path does not visit agent $i$ since $i$ is not on the path from $r$ to $r^{\prime}$, and also $i$ is not located on the cycle between $r^{\prime}$ and $j$ since $i$ is found on the cycle right after agent $j$. Hence, $r \in N_{i}\left(\hat{g}_{-i j}\right)$, implying (3). Hence we conclude that $(j, i)$ is beneficial in $\hat{g}$.

Proposition 1 Let the payoff function be given by (1) where link costs are ownerhomogeneous, i.e. $c_{i j}=c_{i}$ for all $i, j \in N$. Then a Nash network always exists.

Proof Consider the following sequence of networks:

Step 0: Let $t=1$, and $g_{t}=\emptyset$;

Step 1: If $g_{t}$ is Nash then STOP.

Step 2: Let agent $i$ perform a best answer in $g_{t}$, with as many links as possible, and let $g_{t+1}$ be the resulting network;

Step 3: Let $g_{t+2}=\hat{g}_{t+1}$;

Step 4: Let $t=t+2$, and return to Step 1 .

(For convenience we write $\hat{g}_{t+1}$ instead of $\widehat{g_{t+1}}$.)

Let $i$ be an agent who applies a best response $B$ to $g_{t}$ with as many links as possible, obtaining $g_{t+1}$. Suppose that $g_{t}$ is proper and beneficial. We prove that (i) $N_{i}\left(g_{t+1}\right) \supset N_{i}\left(g_{t}\right)$, (ii) $\hat{g}_{t+1}$ is beneficial, and (iii) $\hat{g}_{t+1}$ is proper.

(i) Let $j \in N_{i}^{d}\left(g_{t}\right)$, and suppose $j \notin B$. Since $g_{t}$ is proper it follows that agent $j$ in $\left(g_{t}\right)_{-i}^{j}$ is the unique agent who observes all agents in $\left(g_{t}\right)_{-i}^{j}: N_{j}\left(\left(g_{t}\right)_{-i}^{j}\right)=$ $\operatorname{Car}\left(\left(g_{t}\right)_{-i}^{j}\right)$. This shows that whenever $\operatorname{Car}\left(\left(g_{t}\right)_{-i}^{j}\right) \cap B \neq \emptyset$, then $j \in B$. Since we assumed $j \notin B$, it follows that $\operatorname{Car}\left(\left(g_{t}\right)_{-i}^{j}\right) \cap B=\emptyset$, so that

$$
\begin{aligned}
\pi_{i}\left(g_{t+1} \cup\{(j, i)\}\right) & =\pi_{i}\left(g_{t+1}\right)+\pi_{i}\left(\left(g_{t+1}\right)_{-i j}\right) \\
& =\pi_{i}\left(g_{t+1}\right)+\pi_{i}\left(\left(g_{t}\right)_{-i j}\right) \geq \pi_{i}\left(g_{t+1}\right) .
\end{aligned}
$$

The latter inequality is due to the beneficiality of the links in $g_{t}$. Since the best response $B$ is chosen as large as possible, and the action $B \cup\{j\}$ is at least as good as $B$, we arrive at a contradiction. Hence,

$$
N_{i}^{d}\left(g_{t}\right) \subseteq B
$$

Since $B$ is a strict improvement, we have $N_{i}^{d}\left(g_{t}\right) \subset B$, implying $N_{i}\left(g_{t+1}\right) \supset$ $N_{i}\left(g_{t}\right)$.

(ii) By (4) it follows that $g_{t} \subseteq g_{t+1}$, and this proves the beneficiality of the links $(j, i) \in g_{t}$ in $g_{t+1}$. As for the added links $(j, i)$, with $j \in B \backslash N_{i}^{d}\left(g_{t}\right)$ we observed already (see (2)) that these links are beneficial in $g_{t+1}$. So, $g_{t+1}$ is beneficial, and because of Lemma 3 , we conclude that $\hat{g}_{t+1}$ is beneficial. 
(iii) To show that $\hat{g}_{t+1}$ is proper, by Lemma 1 we only need to check the outdegrees of the agents outside $D\left(g_{t+1}\right)$ in $g_{t+1}$. Furthermore, only the outdegree of the agents in $B \backslash N_{i}^{d}\left(g_{t}\right)$ are raised by 1 , with respect to the outdegree in the proper network $g_{t}$. Therefore, we only need to check that the agents in $B \backslash\left(N_{i}^{d}\left(g_{t}\right) \cup D\left(g_{t+1}\right)\right)$ have outdegree 0 in $g_{t}$.

Consider an agent $j \in B \backslash N_{i}^{d}\left(g_{t}\right)$, and suppose that his outdegree in $g_{t}$ is 1 . Then it is easily checked that $j$ is necessarily located on the cycle of $g_{t}$, i.e. $j \in D\left(g_{t}\right)$. Observe that $D\left(g_{t}\right)$ is a subset of $D\left(g_{t+1}\right)$, so that we conclude that $j \notin B \backslash\left(N_{i}^{d}\left(g_{t}\right) \cup D\left(g_{t+1}\right)\right)$. Hence, all agents in $B \backslash\left(N_{i}^{d}\left(g_{t}\right) \cup D\left(g_{t+1}\right)\right)$ have outdegree 0 in $g_{t}$.

Observe that we started with a beneficial, proper network. By Lemma 2 and part (i) we conclude that the total number of observed agents of the constructed networks does not decrease, and strictly increases when Step 2 is performed. The procedure has therefore to terminate with a network that is Nash. Observe that this network is also beneficial and proper.

Open Access This article is distributed under the terms of the Creative Commons Attribution Noncommercial License which permits any noncommercial use, distribution, and reproduction in any medium, provided the original author(s) and source are credited.

\section{References}

Bala, V., Goyal, S.: A non-cooperative model of network formation. Econometrica 68, 1181-1229 (2000)

Billand, P., Bravard, C., Sarangi, S.: Existence of Nash networks in one-way flow models. Econ Theory (2007). doi:10.1007/s00199-007-0303-y

Derks, J., Kuipers, J., Tennekes, M., Thuijsman, F.: Existence of Nash networks in the one-way flow model of network formation. In: Neogy, S.K. et al. (eds.) Modeling, Computation and Optimization, World Scientific (2008a, forthcoming)

Derks, J., Kuipers, J., Tennekes, M., Thuijsman, F.: Local dynamics in network formation. Tech. rep., Maastricht University, Department of Mathematics (2008b)

Galeotti, A.: One-way flow networks: the role of heterogeneity. Econ Theory 29, 163-179 (2006)

Galeotti, A., Goyal, S., Kamphorst, J.: Network formation with heterogeneous players. Games Econ Behav 54, 353-372 (2006)

Haller, H., Kamphorst, J., Sarangi, S.: (Non-)existence and scope of Nash networks. Econ Theory 31, 597604 (2007)

Haller, H., Sarangi, S.: Nash networks with heterogeneous links. Math Social Sci 50, 181-201 (2005) 